\title{
2D Mapping of Bonding Changes at Peroskite Oxide Interfaces and Around Defects
}

\author{
L. Fitting Kourkoutis, ${ }^{*}$ J. H. Lee, ${ }^{*}$ J. H. Song, $* * * * * * *$ H. Y. Hwang,********* D. G. Schlom,** \\ and D. A. Muller* \\ * School of Applied and Engineering Physics, Cornell University, Ithaca, NY 14853 \\ ** Dept. of Materials Science and Engineering, Cornell University, Ithaca, NY 14853 \\ *** Dept. of Advanced Materials Science, University of Tokyo, Kashiwa, Chiba 277-8561, Japan \\ **** Dept. of Physics, Chungnam National University, Daejeon 305-764, Korea \\ ***** Japan Science and Technology Agency, Kawaguchi, 332-0012, Japan
}

Scanning transmission electron microscopy (STEM) in combination with electron energy loss spectroscopy (EELS) has proven to be a powerful technique for characterizing buried structures, such as thin films, interfaces and defects in crystals. Today, atomic-resolution is routinely obtained, and with the correction of electron-optical aberrations sub-Ångström imaging is now possible [1, 2]. Resolution improvement is, however, not the only benefit of aberration correction. By correcting the electron-optical aberrations up to third- or even fifth-order the probe-forming aperture can be opened up and for the same probe size the beam current can be greatly increased. With a matching increase in collection angle, a single core-loss EELS spectrum with a signal-to-noise ratio that allows the extraction of bonding information, can be recorded in milliseconds; and a full two dimensional (2D) map in under a minute [3]. Aberration correction has therefore allowed spatially resolved EELS to enter the era of 2D imaging of composition and bonding at the atomic-scale.

Using these techniques, microscopic inhomogeneities and atomic-scale interdiffusion and bonding changes can now readily be characterized. Here, we demonstrate this for perovskite oxide thin films and heterostructures. As an example, atomic resolution 2D mapping of bonding changes at the $\mathrm{EuTiO}_{3} / \mathrm{DyScO}_{3}$ interface through spectroscopic imaging (SI) is shown in Fig. 1. We find an increased Eu valence in one atomic layer at the interface. Similarly, the reduction of the Ti valence in a Eu-deficient defect in the $\mathrm{EuTiO}_{3}$ film is shown in Fig. 2.

How does the presence of interfaces and defects affect the physical properties of the complex oxide heterostructure? Bulk $\mathrm{La}_{0.7} \mathrm{Sr}_{0.3} \mathrm{MnO}_{3}$ (LSMO) is a conducting ferromagnet at room temperature. However, in manganite/titanate tunnel junctions interface spin and charge modulations are thought to create an interfacial dead layer, thus fundamentally limiting the use of this material in small dimensions. Using spectroscopic imaging at the atomic scale we show instead that the degradation of the magnetic properties of atomically-thin $\mathrm{LSMO}_{/} \mathrm{SrTiO}_{3}$ multilayers correlates with the presence of extended defects in the LSMO layers and a high degree of intermixing at the interfaces. When these extrinsic defects are eliminated, metallic ferromagnetism at room temperature can be stabilized in ultrathin manganite layers.

[1] M. Haider et al., Nature 392 (1998) 768.

[2] P.E. Batson et al., Nature 418 (2002) 617.

[3] D.A. Muller et al., Science 319 (2008) 1073.

[4] This research was primarily supported by the Cornell Center for Materials Research (NSF No. DMR0520404 and No. IMR-0417392). 

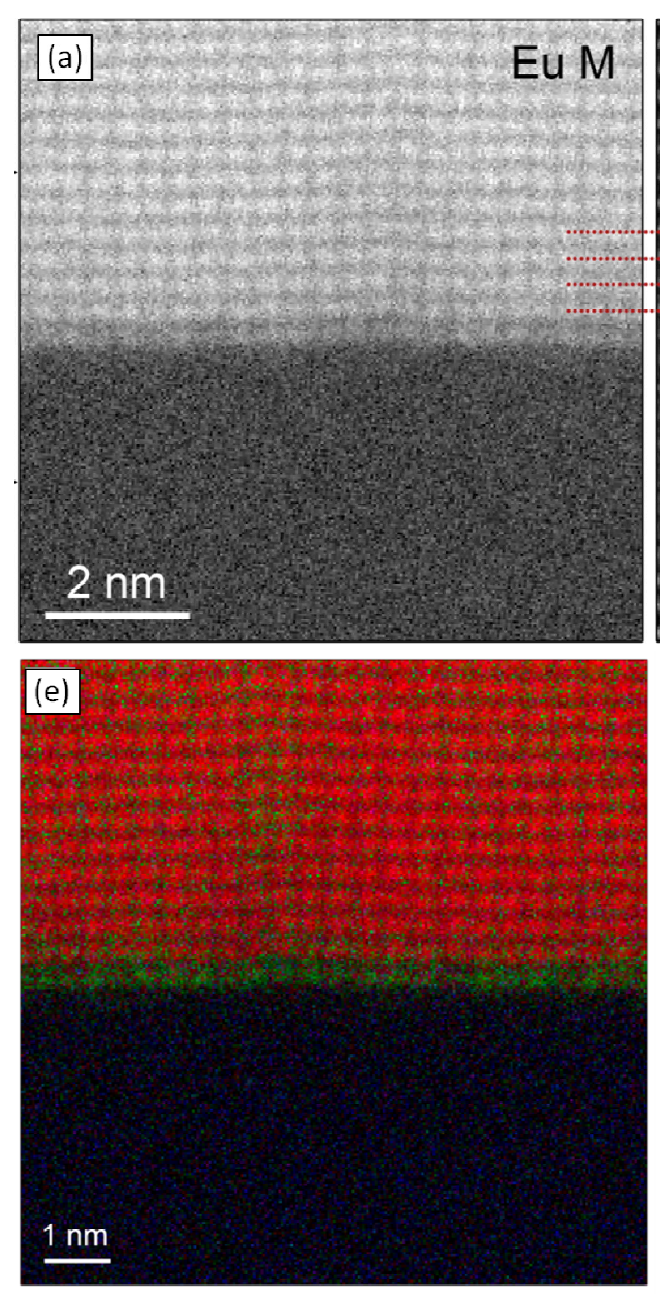
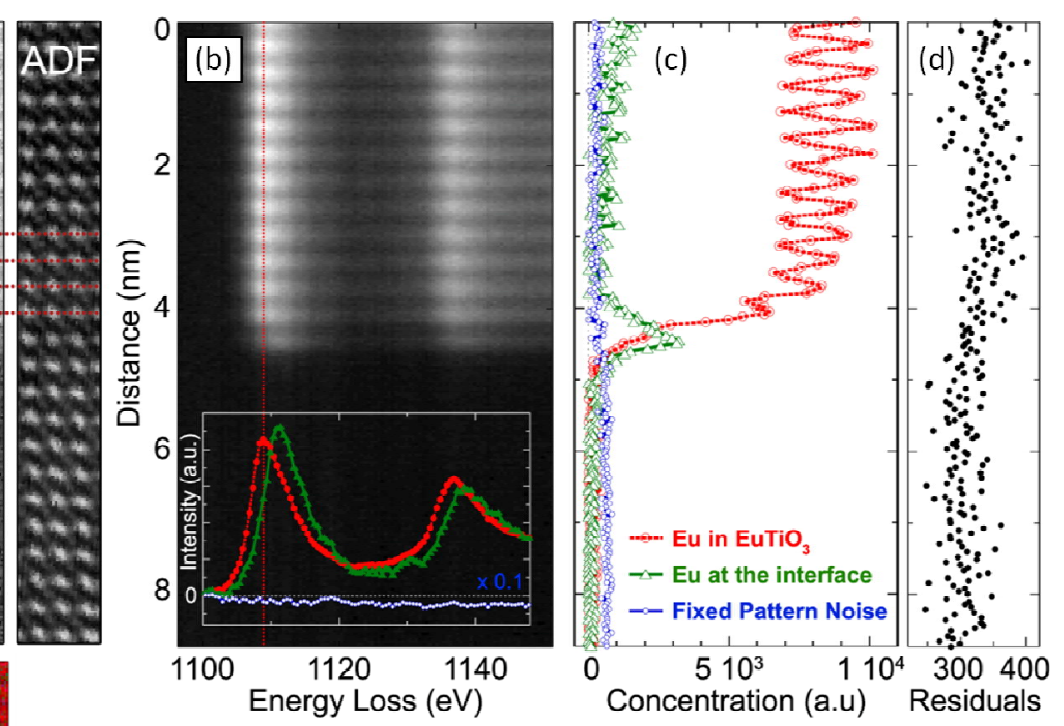

FIG. 1. Mapping bonding changes at the $\mathrm{EuTiO}_{3} / \mathrm{DyScO}_{3}$ interface through spectrum imaging (SI). (a) Eu elemental map showing a reduced Eu concentration at the interface. A part of the simultaneously recorded HAADF image is shown for comparison on the right. (b) Evolution of the horizontally averaged Eu-M edge fine structure across the interface and the three components extracted using MCR methods. (c) The three-component fit to the experimental data shows the increase in Eu valence at the interface. (e) Three-component fit to the full SI demonstrating 2D mapping of bonding changes with atomic resolution.
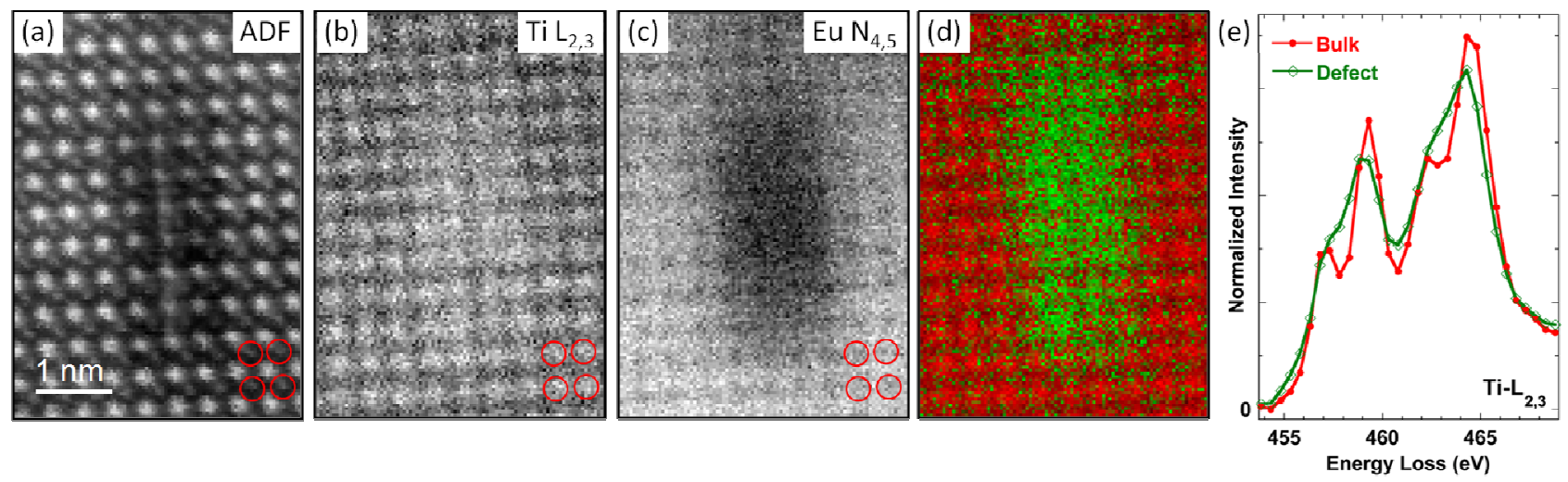

FIG. 2. 2D mapping of composition and bonding of a defect in $\mathrm{EuTiO}_{3}$. (a) ADF STEM image of the defect recorded simultaneously with the 90x127 pixel spectroscopic image. (b) Ti-L and (c) Eu-N elemental maps obtained from the same SI reveal the defect to be Eu deficient. Using MCR methods two statistically significant components to the Ti-L edge were extracted; the first one corresponding to $\mathrm{Ti}^{4+}$ in bulk $\mathrm{EuTiO}_{3}$ and a second one showing a reduced Ti valence (e). (d) Two-component fit to the Ti-L SI using the reference spectra from (e). The concentrations of the two components are shown in red (bulk) and green (defect). Using spectroscopic imaging the defect is shown to be Eu deficient with a reduced $\mathrm{Ti}$ valence compared to $\mathrm{Ti}^{4+}$ in bulk $\mathrm{EuTiO}_{3}$. 